Cahiers de civilisation médiévale

\title{
Enrico Pisano, Liber Maiorichinus de gestis Pisanorum illustribus
}

\section{Kristjan Toomaspoeg}

\section{OpenEdition}

1 Journals

Édition électronique

URL : https://journals.openedition.org/ccm/6818

DOI : $10.4000 / \mathrm{ccm} .6818$

ISSN : 2119-1026

Éditeur

Centre d'études supérieures de civilisation médiévale/Université de Poitiers

\section{Édition imprimée}

Date de publication : 1 janvier 2021

Pagination : 72-74

ISBN : 978-2-490783-08-3

ISSN : 0007-9731

\section{Référence électronique}

Kristjan Toomaspoeg, "Enrico Pisano, Liber Maiorichinus de gestis Pisanorum illustribus », Cahiers de civilisation médiévale [En ligne], 253 | 2021, mis en ligne le 01 mars 2021, consulté le 02 mars 2023. URL : http://journals.openedition.org/ccm/6818; DOI : https://doi.org/10.4000/ccm.6818

\section{c) (†) $\ominus$}

Creative Commons - Attribution - Pas d'Utilisation Commerciale - Pas de Modification 4.0 International - CC BY-NC-ND 4.0

https://creativecommons.org/licenses/by-nc-nd/4.0/ 
Enrico Pisano, Liber Maiorichinus de gestis Pisanorum illustribus, Giuseppe Scalia (éd.), Alberto Bartola (collab.) et Marco Guardo (trad.), Florence, Sismel (Edizione nazionale dei testi mediolatini d'Italia, 44), 2017.

Le poème connu Liber Maiorichinus est sans doute l'une des meilleures sources pour comprendre le rôle tenu par les Pisans durant les $\mathrm{XI}^{\mathrm{e}}$ et $\mathrm{XII}^{\mathrm{e}} \mathrm{s}$. et l'importance politique, militaire et économique de la ville, dont cette œuvre décrit le moment culminant, l'expédition militaire contre les îles Baléares dans les années 1113-1115. Les anciens rivaux de la ville toscane, comme Amalfi, ne parvenaient alors plus à freiner son expansion, tandis que les nouveaux ennemis, plus précisément Gênes, puis Florence, ne constituaient pas encore une menace radicale et immédiate. Comme l'indique bien l'introduction de la présente édition, la conquête des Baléares doit être reliée avec une série d'expéditions navales des Pisans contre les Musulmans de la Méditerranée, en réaction aux incursions arabes des années 1104-1105 et 1111-1112. Les Pisans combattaient à Messine et Reggio en 1005, en Sardaigne en 1015-1016, à Bona en Afrique en 1034, à Palerme en 1064 et, à la fin du siècle, dans le cadre de la première croisade, en Terre sainte. La campagne de 1113-1115 fut conduite par les Catalans de Raymond-Bérenger III de Barcelone et d'autres alliés, comme Lucques, et fut marquée par les sièges d'Ibiza et de Majorque, dévastées par les troupes chrétiennes. La victoire des Pisans et des Catalans se limita toutefois avec l'anéantissement de la puissance économique et militaire des Musulmans des Baléares - déjà affaiblis après le passage des troupes de Sigurd I ${ }^{\text {er }}$ de Norvège quelques années auparavant - et il faut attendre le siècle suivant pour la conquête définitive des îles. Pourtant, la victoire pisano-catalane sur un ennemi considéré comme puissant et redoutable a bénéficié d'un formidable écho, en contribuant à la renommée de Pise, consolidée plus tard lorsqu'Innocent II, durant le schisme d'Anaclète II, la choisit comme propre résidence et lorsque Bernard de Clairvaux en fit les éloges.

Comme le notent justement les éditeurs du Liber Maiorichinus, les victoires des Pisans ont été perçues dans l'historiographie comme des expéditions aux fins exclusivement (ou presque) commerciales. Pourtant, même une lecture superficielle de l'œuvre nous indique la complexité du phénomène. Outre les indéniables raisons liées à la nécessité d'assurer la sécurité et de favoriser l'expansion de la navigation dans la Méditerranée, le Liber Maiorichinus nous emmène dans le contexte spirituel du moment, peu après la première croisade. Son auteur insiste sur le rôle tenu par l'archevêque de Pise, Pierre, qui est allé à Rome, auprès du pape, pour "prendre la croix». Pour Giuseppe Scalia, cette expédition pourrait bien être citée parmi les «croisades en occident» dont elle possède toutes les caractéristiques. Dans le même temps, le fond idéologique de la guerre était complété par l'orgueil et l'esprit communal de Pise, ville porteuse d'une nouvelle Romanitas 
affrontant les «Barbares». Le Maiorichinus représente donc deux tendances historiographiques différentes, «l'épique des croisades» et le "genre épique historique citadin ». Il est bien question d'une œuvre destinée à célébrer la ville de Pise, qui ne reste toutefois pas générique, mais fournit des informations très détaillées et concrètes, vérifiables à travers les autres sources existantes : son auteur est un participant direct à l'expédition et il décrit donc ce qu'il a vu avec ses propres yeux, peu de temps après les événements (sans doute avant 1126).

L'œuvre est composée de 3544 hexamètres et est divisée en huit livres. Le premier livre explique les motivations de l'emprise, donc les méfaits du «tyran» qui régna aux Baléares (Nāṣir ad-Dawla), puis il décrit les préparatifs politiques et militaires de l'expédition et le débarquement des Pisans, au mois d'août 1113 ; le deuxième raconte l'arrivée et le séjour des combattants en Catalogne et en Provence; le troisième parle des négociations avec le souverain des Baléares, l'arrivée de la flotte des Pisans à Ibiza en mars 1114 ; le quatrième est entièrement consacré à la prise d'Ibiza en août 1114; les cinquième, sixième, septième et huitième traitent du siège de Majorque, jusqu'à la victoire finale, en février 1115 et le retour au pays des Pisans.

Les éditeurs définissent ce poème comme une «chronique en vers» écrite par un homme de l'Église pourvu d'une excellente connaissance des auteurs classiques de l'Antiquité, aussi bien grecs que latins, avec une grande habileté de langage et de style. On note en particulier les nombreuses fois où l'auteur puise dans Virgile, surtout dans l'Énéide. Il est aussi bien un habile versificateur, qui se perd peu dans les anomalies prosodiques, qu'un bon connaisseur de la rhétorique. En réalité, cette édition est la première qui permet une identification convaincante de l'auteur du Liber Maiorichinus, grâce aux recherches menées par G. Scalia. Depuis les temps de Ferdinando Ughelli, au XVII ${ }^{\mathrm{e}}$ s., on avait attribué l'œuvre à un Laurent de Vérone, chapelain de l'archevêque de Pise, puis on avait proposé comme nom de l'auteur non Vérone mais Verne ou Varna, puis en 1893 Serafino Marchetti avait avancé le nom d'un autre ecclésiastique, le piévan Henri. Ensuite, la théorie officielle proposa Laurent comme continuateur du travail d'Henri, jusqu'au moment où G. Scalia lui-même, dans les années 1950, prouva que l'œuvre avait été écrite par un seul auteur. Il s'agit en effet du prêtre Henri, piévan de Calci, auquel on peut attribuer aussi quelques textes épigraphiques monumentaux. Laurent de Vérone, augustinien, est en revanche connu bien plus tard, au XIV ${ }^{\mathrm{e}}$ s., et il est bien probable qu'il ait été le propriétaire du manuscrit du Liber Maiorichinus.

Cette œuvre est connue à travers quatre manuscrits toujours conservés dont le plus important est à Pise, à la Biblioteca Universitaria, le ms. 723, qui contient le texte dans la version de la première moitié du $\mathrm{XII}^{\mathrm{e}} \mathrm{s}$., tandis qu'à Florence, à la Biblioteca Medicea Laurenziana, Rediano pour le ms 202 nous trouvons le texte de la seconde moitié du XIV ${ }^{\mathrm{e}} \mathrm{s}$., à Londres, à la British Library, Additional le ms. 10315 de la fin du XIV ou du début du XV ${ }^{\mathrm{e}} \mathrm{s}$., et à Florence, dans la Biblioteca Nazionale Centrale, Magliabechiano le ms. XXV.479, du Xvi ${ }^{e}$ s. L'editio princeps est celle de Ferdinando Ughelli, dans le troisième volume de la première édition de Italia Sacra, en 1645, reprise sans critiques en 1725 par Ludovico Antonio Muratori dans Rerum Italicarum Scriptores (vol. VI), rééditée en 1841 par Joaquim M. Bover, puis de nouveau (suivant toujours F. Ughelli) par l'abbé Migne dans Patrologia Latina (vol. CLXIII) en 1854. L'édition jusqu'à ce moment-là plus critique est celle-ci réalisée en 1904 sous la direction de Carlo Calisse pour les Fonti per la Storia d'Italia, la plus récente étant en revanche celle espagnole de Jaume Juan Castelló, de 1996.

La présente édition est la seule qui puise dans tous les manuscrits connus, en y ajoutant aussi les informations disponibles sur quatre autres aujourd'hui disparus. Elle est due avant tout à l'érudition de G. Scalia, l'un des membres fondateurs de la Société internationale pour l'étude du Moyen Âge latin (Sismel), qui a écrit dès 1954 sa thèse de perfectionnement auprès de l'École normale supérieure de Pise sur le Liber Maiorichinus et a étudié pendant de longues années des sources narratives pisanes, dont il a publié, par ex., en 2010 les Gesta triumphalia per Pisanos facta. Les autres éditeurs sont Alberto Bartola (Université de Rome «La Sapienza»), spécialiste de langue et littérature latine médiévale et humaniste et Marco Guardo, directeur de la Bibliothèque de l'Accademia Nazionale dei Lincei e Corsiniana à Rome.

L'édition est précédée d'une longue et exhaustive introduction qui traite respectivement du contexte historique du poème, de sa structure et de sa composition, ainsi que l'identification de son auteur, la tradition manuscrite de l'œuvre, mais aussi les diverses versions du texte conservées et les différences entre elles, l'historique des éditions du Liber Maiorichinus et, enfin, une brève description des critères de la présente publication. L'introduction est suivie de la liste de la bibliographie utilisée, 1500 titres, peu ou prou. 
L'édition proprement dite, sans compter les commentaires, occupe 271 pages du volume (p. 184-544). Elle consiste en une reproduction analytique du texte en latin, sur la page de gauche, épaulée de la traduction italienne, à droite. Les éditeurs ont dû résoudre une série de problèmes d'ordre technique : avant tout, ils ont affronté la nécessité d'indiquer au lecteur les variations du texte et les différences entre les diverses versions, en insérant par conséquent en bas de page de gauche les notes contenant ces précisions. Le texte ne se base, par ailleurs, pas exclusivement sur la version du manuscrit de la Bibliothèque universitaire de Pise, mais en corrige certaines erreurs, en s'appuyant sur d'autres sources. Puis, les éditeurs ont rencontré la nécessité de fournir au lecteur les informations sur les très nombreuses citations des œuvres classiques - effectuées par l'auteur du Liber Maiorichinus, sans toutefois indiquer ses sources - ceci a été résolu à d'une double manière : en insérant ces informations en bas de la page de la traduction à droite, et en fournissant à la fin du volume un long index de loci similes. Il s'est aussi avéré nécessaire de munir le lecteur d'un instrument de comparaison avec l'édition de C. Calisse, de 1904, pour faire correspondre les citations de celle-ci avec la présente édition : pour cela, les annexes du volume contiennent aussi un tableau de correspondances spécifique (rédigé par Giuseppe Castello).

Une simple édition du texte du Liber Maiorichinus n'aurait offert qu'une satisfaction partielle, vu le grand intérêt du public cultivé des non-latinistes pour les sources narratives du Moyen Âge : en témoigne la longue série de chroniques et autres textes traduits en italien pendant ces dernières années. La traduction d'une œuvre aussi complexe par son style et sa morphologie a sans doute mis les éditeurs devant le choix habituel : traduire le poème en vers, en conservant le style et en perdant la précision des détails ou bien en prose, en abandonnant la forme. Sur ce point, les éditeurs ont pris la décision, tout à fait partageable, de traduire le Liber Maiorichinus en vers, mais en en utilisant le verso sciolto (le «vers libre»), sans suivre aucun schéma métrique obligatoire. Cela permet de conserver la précision du texte, sans sacrifier sa forme poétique, aulique. La lecture de la traduction et une comparaison de celle-ci avec le texte latin ne font que renforcer cette opinion. Les noms de personnes et de lieux sont présentés, lorsque cela est possible, dans leur version italienne, sauf les noms arabes, transcrits selon les critères de l'Encyclopedia of Islam.

L'édition du Liber Maiorichinus est suivie d'une longue série de commentaires historiques, géographiques, littéraires et linguistiques approfondis, séparée du texte (p. 457-595), dont il n'est sans doute pas nécessaire de souligner l'utilité. Les indices du volume, rédigées par A. Bartola, nous offrent, outre la liste des citations des classiques, celle des personnes, lieux et choses notables présents dans le Liber Maiorichinus, un utile répertoire des manuscrits et sources d'archive utilisés, la liste bibliographique des auteurs antiques et médiévaux cités et, enfin, celle des auteurs contemporains.

On ne peut que considérer cette édition du Liber Maiorichinus comme un remarquable exemple d'érudition mis au service du public. À la différence de quelques autres publications récentes de sources narratives, réalisées un peu «à la hâte», celle-ci est le fruit de décennies de travail de recherche non seulement linguistique et codicologique, mais aussi historique, en équilibre parfait entre l'œuvre et son contexte.

Kristjan ToOmASPOEG Université du Salento, Lecce 